\title{
Las Cumbres Observatory: Networking People and Telescopes for Science and Education
}

Edward Gomez ${ }^{1 *}$

${ }^{1}$ Los Cumbres Observatory

*Corresponding author: egomez@lco.global

\section{Introduction}

Education has been one of the core themes of Las Cumbres Observatory (LCO) since it first began operating telescopes in 2005. Since 2014, to maximize the effectiveness and reach of $\mathrm{LCO}$ we have been working with 8 organizations who have excellent track records in providing and fully supporting astronomy education programs. These "education partners" provide structured educational projects for their audience, within which observing and handling data through LCO is an integral component.

During the period 1 June 2016 to 1 June 2017, 1,500 individual education partner accounts observed a total of 75,000 images using LCO. The volume and variety of programs which these images and data enabled, is much more than the LCO education team could have managed, maintained and supported alone. Many of these user accounts are used by teachers leading classes, so the impact of the access to LCO is probably larger than the number of active accounts.

These 8 education partners in 2017 were:

- Our Solar Siblings (Australia)

- Universe in the Classroom (Wales)

- Faulkes Telescope Project (UK)

- University of Hawai'i (USA)

- Universe Awareness (Global)

- McDonald Observatory (USA)

- IASC (Global)
- Hands on Universe (Global).

Based on the experiences of the last few years, we are now ready to open this opportunity to a wider group, by launching an annual call for education partner proposals. With more telescopes than ever in the LCO network, and a new suite of observation scheduling tools, now is the perfect time to expand the education partner program.

\section{What Can LCO Offer an Education Partner?}

LCO has 3 aspects which make it unique as an observatory. Combined these aspects make LCO highly versatile for a large variety of astronomy education projects.

1. Global coverage in Northern and Southern hemispheres. We currently have 7 high quality, professional observatory sites with 20 telescopes spread between them. We have plans to deploy more 0.4-meter and possibly 1-meter telescopes during 2017-2018 (see Fig. 1).

2. A homogeneous network of telescopes, in 3 classes, 2-meter, 1-meter and 0.4-meter, built in-house. Telescopes in each class are identical, as are their instrumentation. Our 2-meter telescopes have imaging cameras and low-resolution spectrographs, our 1-meter telescopes have imaging cameras and fiber-fed high resolution spectrographs, and our 0.4-meter telescopes have imaging cameras. 


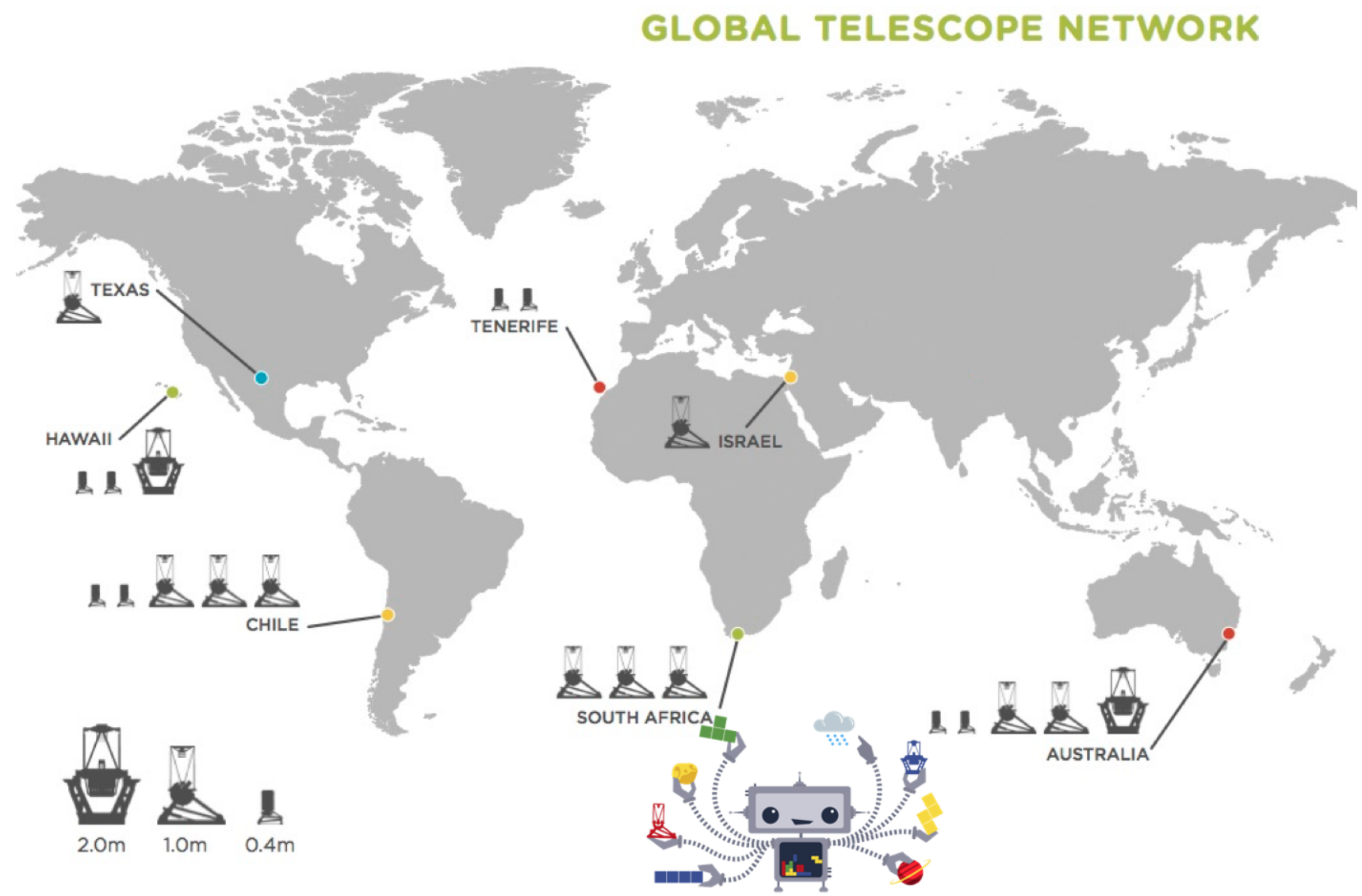

Figure 1. Las Cumbres Observatory global telescope network, showing all telescopes currently deployed (as of June 2017).

3. SEROL, the LCO dynamic, autonomous scheduling software. SEROL (also developed in-house) constantly shuffles observation requests around our network, responding to changes in weather conditions and new requests being made by astronomers and educators from around the world.

\section{Is Your Program a Good Fit?}

We believe in using the right tools for each task. Inevitably these means that some education programs are not suited to using LCO. We cannot provide the type of inspirational experience that looking through a telescope eye piece will provide. To make our observatory efficient, SEROL removes most of the traditional observation planning needed to obtain data. However, there are many advantages to our system, which we believe are assets to educational and research activities. Because of our geographic spread and SEROL's dynamic scheduling, LCO is ideal for studying time-domain astrophysical phenomena.

Time-series and solar system targets are areas which would be tedious to schedule by hand, but Serol's scheduling capabilities make trivial.

For more information about education partner programs, please see the LCO education partner web page (https://lco.global/education/partners/). 